INTESTINE

\title{
Depletion of intestinal resident macrophages prevents ischaemia reperfusion injury in gut
}

\author{
Y Chen, V C H Lui, N V Rooijen, P K H Tam
}

Gut 2004;53:1772-1780. doi: 10.1136/gut.2003.034868

See end of article for authors' affiliations

Correspondence to:

Dr P K H Tam, Division of Paediatric Surgery, Department of Surgery, University of Hong Kong Medical Centre, Queen Mary Hospital, Pokfulam, Hong Kong SAR, China; paultam@hkucc.h́ku.hk

Revised version received 2 May 2004

Accepted for publication

6 May 2004
Background and aims: The cellular and molecular events involved in ischaemia reperfusion (IR) injury are complex and not fully understood. Previous studies have implicated polymorphonuclear neutrophils (PMN) as major inflammatory cells in IR injury. However, anti-PMN antiserum treatment offers only limited protection, indicating that other inflammatory cells are involved. We have therefore investigated the contribution of resident macrophages in IR injury using an IR gut injury model.

Methods: DA rats were divided into sham operation and IR groups. The superior mesenteric artery was clamped for 30, 45, or 60 minutes (ischaemia) followed by 60 minutes of reperfusion. IR injuries were evaluated by histological staining. Expression of early growth response factor 1 (Egr-1), myeloperoxidase (MPO), and proinflammatory cytokines was analysed by immunohistochemistry, reverse transcriptionpolymerase chain reaction, and western blotting analysis. The specific role of macrophages in IR gut injury was also evaluated in resident macrophage depleted rats.

Results: Mucosal sloughing and villi destruction were seen in 45/60 minute and 60/60 minute IR guts. PMN infiltration at the damaged mucosal area was undetectable in $45 / 60$ minute and $60 / 60$ minute IR guts. PMN were localised around the capillaries at the base of the crypts in 60/60 minute IR gut. Obvious PMN infiltration was only observed in damaged villi after three hours of reperfusion. Elevated nuclear Egr-1 immunostaining was localised in resident macrophages at the damaged villi before histological appearance of mucosal damage. Furthermore, resident macrophages at the damaged site expressed MPO. Protein levels of the proinflammatory cytokines RANTES and MCP-1 were increased in IR gut. Depletion of resident macrophages by dichloromethylene bisphosphonate significantly reduced mucosal damage in rat guts after $\mathbb{R}$.

Conclusion: Our findings indicate that resident macrophages play a role in early mucosal damage in $I R$ gut injury. Therefore, macrophages should be treated as a prime target for therapeutic intervention for IR damage.
$\mathrm{T}$ he intestinal mucosa, which lines the lumen of the intestine, acts as a defence barrier against pathogen invasion. Ischaemia reperfusion (IR) causes damage to the intestinal mucosa, producing increased gut permeability and bacterial translocation which can result in systemic inflammatory response syndrome and multiple organ failure. IR injury associated mortality remains high despite advances in patient care for IR injury. ${ }^{1}$ The cellular and molecular events involved in IR injury are complex, involving the production of reactive oxygen species (ROS), reactive nitrogen species, and polymorphonuclear neutrophils (PMN) infiltration. This is accompanied by the release of other moderators such as nitric oxide, platelet activation factor, and phospholipase A2. ${ }^{2}$ Recruitment of PMN into reperfused sites after ischaemia is critical for IR injury in the heart, lung, and liver. $^{3-5}$ Anti-PMN antiserum treatment offers only limited protection against IR injury in the heart. ${ }^{6}$ This suggests that other inflammatory cells are likely to be involved. For example, mast cells have also been suggested to play a role in IR injury, ${ }^{7}$ and anti-complement agent K-76COONa, which prevents mast cell degranulation, has been shown to ameliorate gut IR injury. However, K-76COONa also inhibits other inflammatory cells such as macrophages ${ }^{8}$ and lymphocytes. ${ }^{9}$ Identifying the additional inflammatory cells that contribute to gut IR injury remains a priority for the development of an effective treatment strategy directed towards specific cellular targets.

Macrophages are inflammatory cells, which on activation by bacterial lipopolysaccharide destroy bacteria by the release of cytokines, ROS, induction of myeloperoxidase (MPO) activity, and phagocytosis of bacteria. ${ }^{10}$ Acute inflammation induces PMN infiltration and increases MPO activity in situ, causing local tissue damage. ${ }^{11}$ Elevated MPO activity occurs in an IR injured gut and localises to the ischaemic site shortly after reperfusion and before the appearance of tissue damage. ${ }^{12}$ Activated macrophages exhibit high levels of MPO activity. ${ }^{13}{ }^{14}$ Activation of macrophages mediates the apoptosis of stromal, parenchymal, and other immune cells. ${ }^{15}$ Chloramines, which are catalytic products of MPO, can cause growth arrest and apoptosis in gastric mucosal cells. ${ }^{16} 17$ However, it is still unclear whether the increased MPO activity at the IR site is produced as a result of infiltrated PMN, activated macrophages in situ, or both. ${ }^{18}$ Mucosa damage is an early histological landmark of IR injury to the gut $^{2}{ }^{19}$ which manifests functionally as increased gut permeability. Macrophages mainly locate at the mucosa and smooth muscle layers of the intestine, and are termed either mucosal or muscularis resident macrophages. Hierholzer et al have shown that muscularis resident macrophages release

Abbreviations: IR, ischaemia reperfusion; $P M N$, polymorphonuclear neutrophils; Egr-1, early growth response factor 1; MPO, myeloperoxidase, RT-PCR, reverse transcription-polymerase chain reaction; ROS, reactive oxygen species; ICAM-1, intercellular adhesion molecule 1; MCP-1, monocyte chemoattractant protein 1; SMA, superior mesenteric artery; $\mathrm{Cl}_{2} \mathrm{MBP}$, dichloromethylene bisphosphonate; PBS, phosphate buffered saline; iNOS, inducible nitric oxide synthase; LFA-1, ymphocyte function associated antigen 1 
the proinflammatory cytokine interleukin 6 and directly contribute to decreased gut motility in the IR injured gut. ${ }^{20}$ However, the role of mucosal resident macrophages in intestinal IR injury has not been investigated.

Early growth response factor 1 gene $(E g r-1)$ encodes a transcription factor and controls the development of the central and peripheral nervous systems. ${ }^{21}$ In vitro studies have identified downstream target genes of Egr-1. These include genes encoding tumour necrosis factor $\alpha$, intercellular adhesion molecule 1 (ICAM-1), CD44, platelet derived growth factor $\mathrm{A} / \mathrm{B}$ chain, transforming growth factor $\beta$, and macrophage colony stimulating factor. ${ }^{22}$ Expression levels of Egr-1 are upregulated in the heart, ${ }^{23}{ }^{24}$ lung, $^{22}$ and kidney ${ }^{25} 26$ after IR challenge. These findings indicate that Egr-1 is involved in IR injury in these organs. The involvement of Egr$l$ in IR injury is substantiated by findings that lung injury is less severe and associated with improved survival after IR in Egr-1 knockout mice.22 Production of the proinflammatory cytokine monocyte chemoattractant protein 1 (MCP-1) and adhesion molecule ICAM-1 in IR injured lungs is lower in Egr-1 knockout mice. This probably accounts for the reduced PMN infiltration and mild lung damage seen in lung IR injury in Egr-1 knockout mice.

We hypothesise that resident macrophages play a key role in the initiation of intestinal IR injury. To test our hypothesis, we investigated the cellular and molecular events involved in early gut mucosal damage from IR. We showed in a rat IR gut injury model: (i) the onset of mucosal damage before PMN infiltration; (ii) activation of mucosa resident macrophages in the IR injured gut; (iii) expression of high levels of MPO and Egr-l in activated resident macrophages; and (iv) depletion of intestinal resident macrophages, which prevented IR injury to the gut in rats.

\section{MATERIALS AND METHODS \\ Rat IR gut model}

Six to eight week old DA rats $(\sim 250 \mathrm{~g})$ were divided into sham operation and IR groups. Rats were anaesthetised by intraperitoneal injection ( $45 \mathrm{mg} / \mathrm{kg}$ body weight) of pentobarbital sodium solution (Abbott Lab, Illinois, USA). The superior mesenteric artery (SMA) was occluded by clamping for $30(n=6), 45(n=6)$, or 60 minutes $(n=6)$. A change in colour of the occluded intestine from red to pale immediately after clamping indicated successful induction of ischaemia. After induction of ischaemia, clamps were released and guts were reperfused for 60 minutes. In the sham operation groups, SMA was separated but no occlusion was applied for $30+60$ minutes $(n=6), 45+60$ minutes $(n=6)$, or 60+60 minutes $(n=6)$. The operation site was covered with cotton gauze soaked with saline. Segments of the jejunum $(8-10 \mathrm{~cm})$ of the IR and sham operation rats were collected for RNA, protein, and immunohistochemistry. The study was approved by the ethics committee of the University of Hong Kong.

\section{Macrophages depletion}

Liposome encapsulated dichloromethylene bisphosphonate $\left(\mathrm{Cl}_{2} \mathrm{MBP}\right.$ liposome) or phosphate buffered saline (PBS, control liposome $)^{27}$ was provided by Roche Diagnostics (Mannheim, Germany) and encapsulated in liposomes in Dr Rooijen's laboratory. The $\mathrm{Cl}_{2} \mathrm{MBP}$ or control liposome $(4 \mathrm{ml})$ was injected into the intraperitoneal cavity three days before IR. The liver and jejunum were removed and depletion of macrophages in these organs was verified by immunostaining for resident macrophage. Rats were injected with $\mathrm{Cl}_{2} \mathrm{MBP}$ liposome $(\mathrm{n}=4)$ or control liposome $(\mathrm{n}=4)$, and subjected to 45 minutes of ischaemia and 60 minutes of reperfusion.

\section{Immunohistochemistry and immunofluorescence staining}

Gut specimens were fixed in $4 \%$ paraformaldehyde/PBS (pH 7.2) at $4^{\circ} \mathrm{C}$ for 18 hours, dehydrated in alcohol, cleared in xylene, and embedded in paraffin. Sections $(4 \mu \mathrm{m}$ thickness) of jejunum were mounted onto TESPA (3-aminopropyl-triethoxy-silane; Sigma, St Louis, Missouri, USA) coated microscope glass. Antigen unmasking was performed by heating the sections in $10 \mathrm{mM}$ of citrate buffer $\left(\mathrm{pH} \mathrm{6.2)}\right.$ for 10 minutes at $95^{\circ} \mathrm{C}$. Sections were incubated in $3 \%(\mathrm{v} / \mathrm{v}) \mathrm{H}_{2} \mathrm{O}_{2}$ in methanol to quench endogenous peroxidase activity. Tissue sections were blocked with Dako protein block (Dako A/S, Glostrup, Denmark) and incubated with Egr-1 (Santa Cruz Biotechnology, California, USA; 1:800), EDl (Serotec; 1:100), ED2 (Serotec, Kidlington, Oxford, UK; 1:200) ICAM-1 (Santa Cruz Biotechnology; 1:100), or rat PMN (Accurate, New York, USA; 1:10 000) antibodies in $50 \mathrm{mM}$ Tris buffer $(\mathrm{pH} 7.4)$ containing $1 \%$ bovine serum albumin (USB Corporation, Ohio, USA) at $37^{\circ} \mathrm{C}$ for $40 \mathrm{~min}$ utes or $4^{\circ} \mathrm{C}$ for 16 hours. EDl antibody recognises a cytoplasmic antigen in monocytes and infiltrating macrophages, and is used as a pan macrophage marker. ${ }^{28}$ ED2 antibody, which recognises a membrane bound antigen similar to human CD163, has been commonly used as a specific maker for resident macrophages. ${ }^{28}{ }^{29}$ Signal amplification and detection were performed with the Dako EnVision System, HRP. Sections were counterstained with haematoxylin, dehydrated in ethanol, cleared in xylene, and mounted with DPX mounting medium (BDH Laboratory Supplies, Poole, Dorset, UK). Immunofluorescence staining was performed by incubating FITC and TRITC conjugated secondary antibodies on sections and mounting them in DAPI containing Vectashield mounting medium (Vector Laboratories, California, USA). Immunofluorescent stained sections were analysed with a fluorescence microscope (Nikon Eclipse E600). Three jejunum sections of each rat in different treatment groups were analysed for histology and immunohistochemistry. Rats of the same group showed similar histology and immunostaining pattern. Representative pictures of haematoxylin/eosin or immunostained sections of each group were shown for comparison.

\section{Quantitative analysis of macrophages and PMN in gut} Numbers of ED2 and ED1 immunopositive cells were counted from 10 randomly selected high power $(400 \times)$ fields of jejunum sections. The ratio of ED2 to ED1 immunopositive cells was tabulated under different treatments. Egr-1 immunopositive cells were counted and separated into two groups: cells showing cytoplasmic Egr-1 staining and cells showing nuclear Egr-1 staining. A minimum of three sections of each rat was examined and 10 randomly selected high power $(400 \times)$ fields of each gut section were examined. Two observers counted the sections separately, and the results were averaged and tabulated.

Table 1 Primers used for the polymerase chain reactions

\begin{tabular}{ll}
\hline Primer & Sequence \\
\hline Egr-1: sense & 5'-CCG GT GCC TCC CAT CAC CTA-3' \\
Egr-1: antisense & 5'-GAG CGA AGG CTG CTG GGT ACG-3' \\
MPO: sense & 5' CCG CAG GAC AAG TAT CGA A 3' \\
MPO: antisense & 5' ATC TGG TIG CGA ATG GTA ATG 3' \\
$\beta$-actin: sense & 5'-TGG AAT CCT GTG GCA TCC ATG AAA C-3' \\
$\beta$-actin: antisense & 5'-ACC ATC AAG GCA CAG CAA CTC-3'
\end{tabular}

Egr-1, early growth response factor $1 ; M P O$, myeloperoxidase. 


\section{Quantitative analysis of PMN in gut}

Numbers of PMN (immunopositive for anti-PMN antibody) were counted from 10 randomly selected high power $(400 \times)$ fields of jejunum sections, and three sections of each rat were examined. Two observers counted the sections separately, and the results were averaged and tabulated.

\section{Quantitative analysis of mucosal damage}

Sloughing of villi tips ${ }^{2}$ was used as a histological criterion of IR induced mucosal damage in $\mathrm{Cl}_{2} \mathrm{MBP}$ or control liposome treated rats. Five randomly selected low power $(100 \times)$ fields of gut mucosa sections were examined, and the numbers of damaged villi and the total number of villi were recorded. Three sections from each rat were examined. Two observers counted the sections separately, and the results were averaged and tabulated.

\section{Reverse transcription-polymerase chain reaction (RT-PCR)}

Expression of Egr-1 and $\beta$-actin was analysed by RT-PCR. Full thickness gut wall was used for total RNA isolation using Trizol (Invitrogen, Carlsbad, California, USA). First strand cDNA was synthesised from $1 \mu \mathrm{g}$ of total RNA using oligo $\mathrm{d}(\mathrm{T})_{12-18}$ as a reverse primer and SuperScript First-strand synthesis System (Invitrogen). PCR was performed in $25 \mu \mathrm{l}$ of standard buffer containing primers ( $1 \mu \mathrm{M}$ each; table 1$)$, cDNA $(3 \mu \mathrm{l}), 0.25$ units of AmpliTaq Gold (Applied Biosystems, California, USA), $\mathrm{MgCl}_{2}(1.5 \mathrm{mM})$, and dNTP (0.2 mM each). After initial denaturation $\left(94^{\circ} \mathrm{C}\right.$, three minutes), PCR was performed for 30 cycles for Egr-1, MPO, and $\beta$-actin as follows: 30 seconds at $94^{\circ} \mathrm{C}$; one minute at $60^{\circ} \mathrm{C}$; and one minute at $72^{\circ} \mathrm{C}$ for each cycle. A final extension for 10 minutes at $72^{\circ} \mathrm{C}$ was added. PCR products were analysed by electrophoresis in $1 \%$ agarose gel. Intensities of PCR bands were quantified and relative expression levels of Egr-1 to $\beta$-actin were determined using Scion Image software (Scion Co, Massachusetts, USA).

\section{Western blotting analysis}

Total proteins, and cytoplasmic and nuclear protein fractions were prepared as previously described..$^{30}$ Equal amounts of protein $(50 \mu \mathrm{g}$ or $20 \mu \mathrm{g}$ in the case of nuclear protein) of each sample were separated in $12.5 \%$ sodium dodecyl sulphate polyacrylamide gels before being electrotransferred onto a PVDF membrane (Amersham Bioscience, Hong Kong, China) in a transfer buffer (48 mM Tris, $39 \mathrm{mM}$ glycine, 20\% methanol, $\mathrm{pH}$ 9.2). The membrane was blocked in PBST $(\mathrm{pH} 7.2,0.1 \%$ Tween 20$)$ containing $10 \%(\mathrm{w} / \mathrm{v})$ skimmed milk for one hour before incubation with primary antibodies in PBST against Egr-1 (Geneka Biotechnology, Quebec,

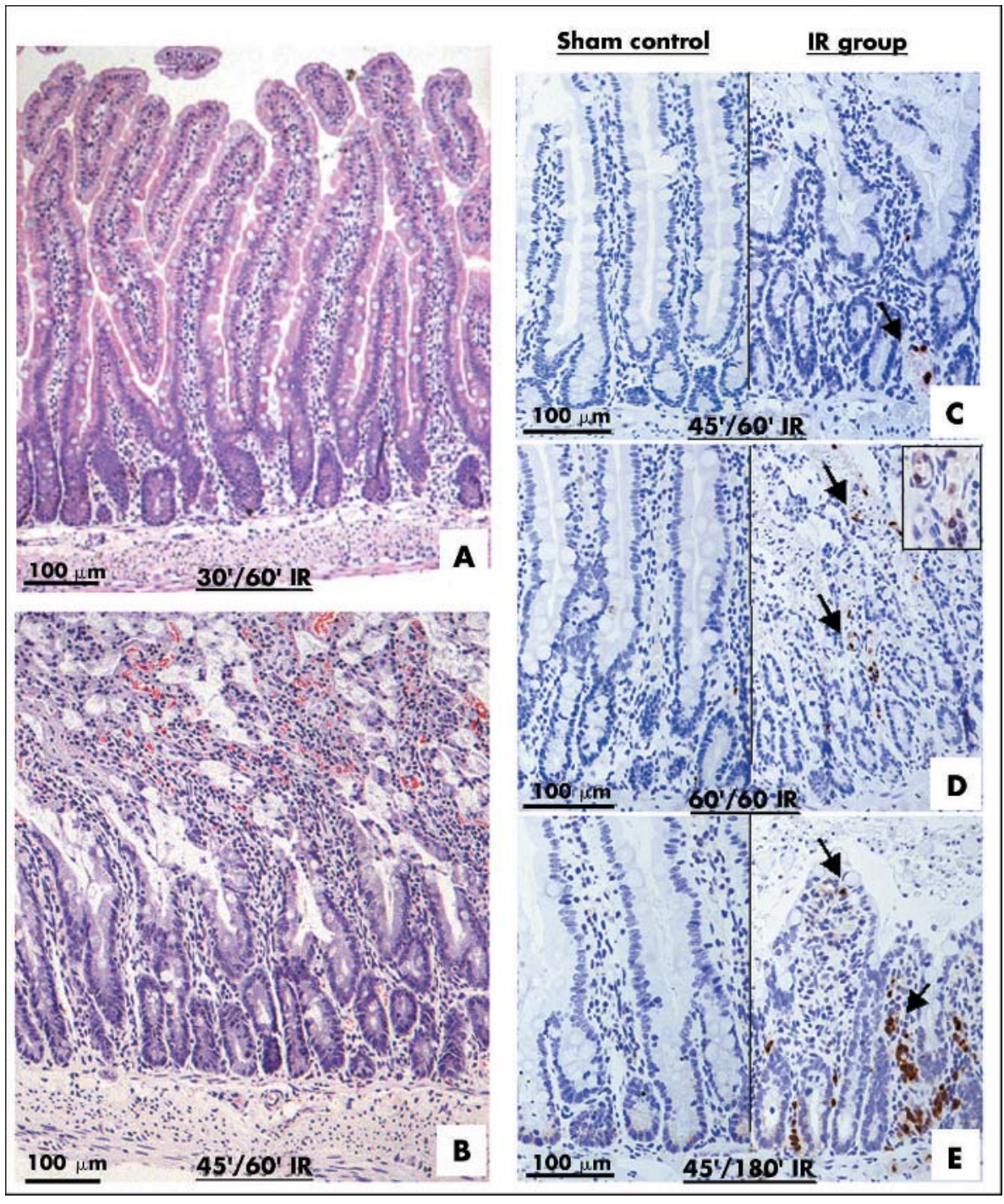

Figure 1 Ischaemia reperfusion (IP) injury of gut occurs before polymorphonuclear neutrophil (PMN) infiltration. Sections of jejunum of different IR groups were stained with haematoxylin and eosin. Clear mucosal damage was observed in the $45 /$ 60 minute $\left(45^{\prime} / 60^{\prime}\right)$ IR gut (B) but not in the $30 / 60$ minute $\left(30^{\prime} / 60^{\prime}\right)$ IR gut (A). Sections of sham controls and IR groups were immunostained with antiPMN antibody (C-E). Few PMN (arrows) were localised in the 45 / 60 minute IR jejunum (C). PMNs were detected near and inside the blood capillaries at the base of the crypt of the 60/60 minute IR jejunum ( $D$; inset). Heavy PMN infiltration was detected in the $45 / 180$ minute $I R$ jejunum (E). 

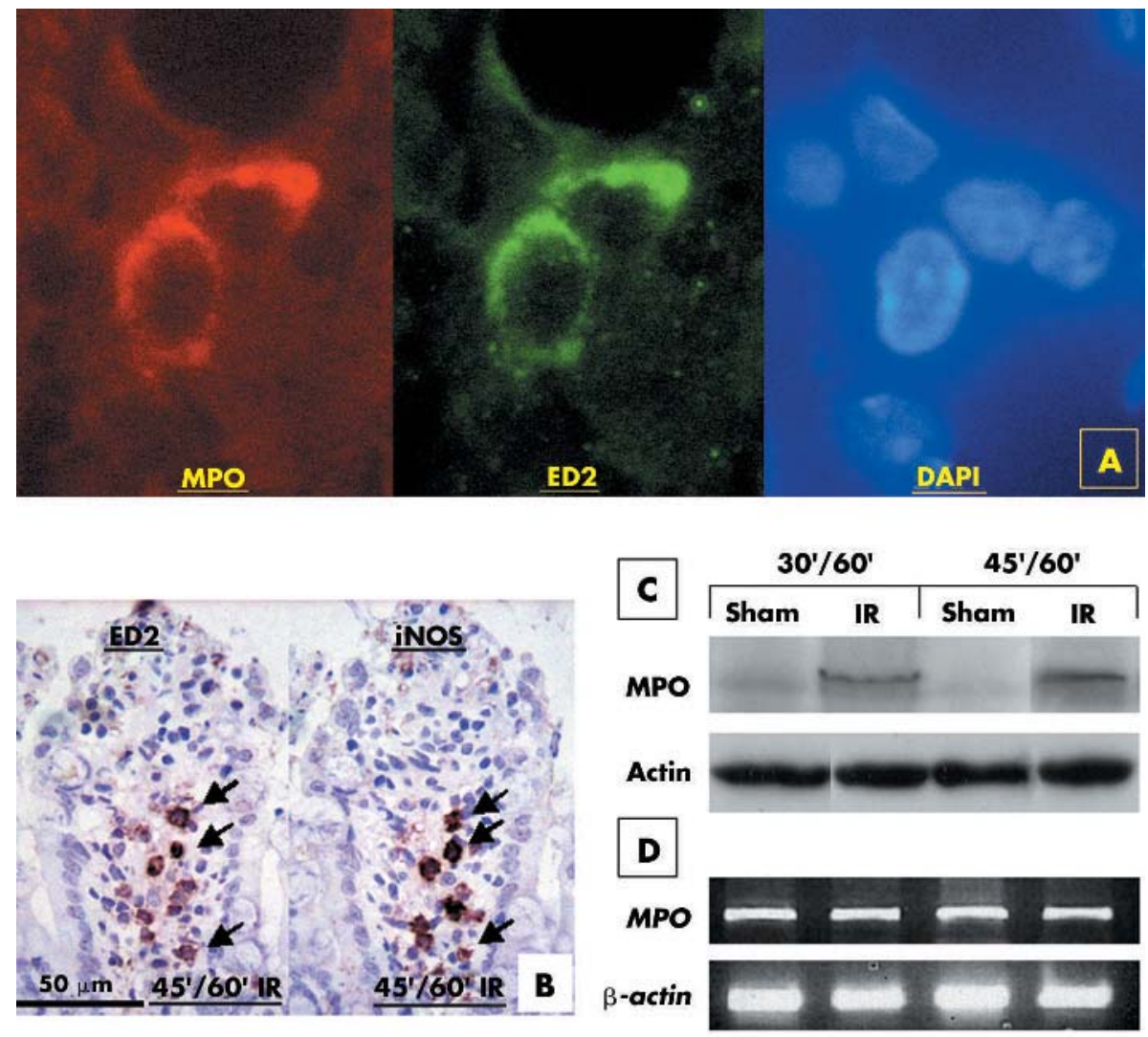

Figure 2 Resident macrophages express myeloperoxidase (MPO) and inducible nitric oxide synthase (iNOS) in ischaemia reperfusion (IR) gut. Sections of $45 / 60$ minute $\left(45^{\prime} / 60^{\prime}\right)$ IR jejunum were immunostained using anti-MPO and ED2 antibodies (A). ED2 immunoreactive resident macrophages were also immunoreactive for MPO. Adjacent sections were immunostained with anti-iNOS antibody and ED-2 antibody (B). ED2 immunopositive were also iNOS positive. Protein extracts of the sham operation and IR jejunum of $30 / 60$ minute $\left(30^{\prime} / 60^{\prime}\right)$ and $45 / 60$ minute $\left(45^{\prime} /\right.$ $60^{\prime}$ ) ischaemic groups were analysed by western blotting (C). Levels of MPO were increased in the 30 minute and 45 minute IR jejunum (IR) but a negligible level of MPO was detected in the sham operation jejunum (sham). MPO mRNA levels were similar between the sham operation and IR jejunum, as revealed by reverse transcription-polymerase chain reaction (D).

Canada; diluted 1:1000), RANTES, ICAM-1, MCP-1 (Santa Cruz Biotechnology; diluted 1:100), or actin (Santa Cruz Biotechnology; diluted 1:2000) for 16 hours at $4^{\circ} \mathrm{C}$. After three PBST washings, blots were incubated with appropriate horseradish peroxidase conjugated secondary antibodies (Zymed, California, USA) in PBST for one hour at ambient temperature. After three PBST washings, signal detection was performed with an ECL kit (Amersham Bioscience).

\section{Statistical analysis}

Means (SEM) of the relative expression of Egr- 1 to $\beta$-actin, ratio of ED2 to EDl immunopositive cells, and ratio of damaged villi to total villi were determined for each group. One way (and non-parametric) ANOVA, with NewmanKeuls multiple comparison test was used (GraphPAD Software, San Diego, California, USA) to evaluate the significance of the differences between different treatments, and a $\mathrm{p}$ value $<0.05$ was considered significant.

\section{RESULTS}

\section{Localisation of PMN in the gut after IR injury}

To define a time point for early intestinal damage after IR, histological analyses were performed on IR guts treated with different periods of ischaemia $(10,30,45,60$, and $120 \mathrm{~min}$ utes) and reperfusion (1,3, 6, and 15 hours). Histological analyses showed that reperfusion after SMA clamping caused IR induced gut damage characterised by mucosal damage, sloughing of the mucosal epithelium, and villi destruction observed in the IR jejunum at 45/60 minutes (fig 1B). Mucosal damage was not evident in the jejunum of either the 30/60 minute IR group (fig lA) or the sham operation group (data not shown). To localise the infiltrated PMN in the IR jejunum, immunohistochemistry using antirat PMN antibody was performed. Only a few PMN were detected in the damaged villi of the jejunum of the $45 / 60$ minute $(0.7$ (0.16):0.65 (0.23); IR:sham) and 60/60 minute IR groups (1.25 (0.28):0.8 (0.2); IR:sham) (fig IC, D). In the $60 / 60$ minute IR gut, PMN were localised near and inside the blood capillaries at the base of the crypt (fig 1D). In contrast, extensive PMN infiltration was detected in damaged villi after prolonged reperfusion (three hours) (28.35 (2.65):0.5 (0.15); IR:sham; p<0.001) (fig lE).

\section{MPO expression and localisation}

Absence of infiltrated PMN in damaged villi of the 45/60 minute IR jejunum prompted us to investigate the role of resident macrophages in mucosal damage. We performed immunofluorescent staining on sections of 45/60 minute IR jejunum using anti-MPO and ED2 antibodies (fig 2A). Expression of MPO overlapped with that of ED2. ED2 immunopositive resident macrophages also stained for inducible nitric oxide synthase (iNOS) and lymphocyte function associated antigen 1 (LFA-1) (fig 2B; data not shown). Expression levels of MPO in the IR jejunum and the 

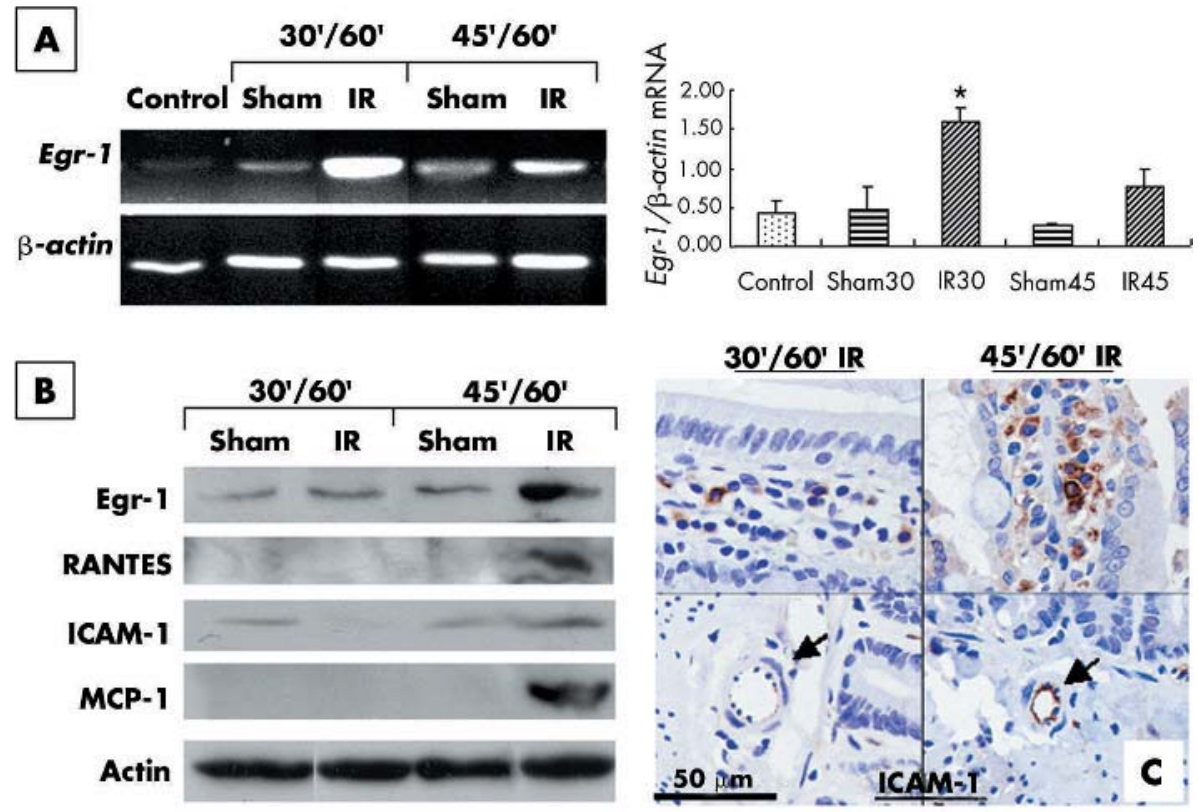

Figure 3 Upregulation of early growth response factor 1 (Egr-1), RANTES, monocyte chemoattractant protein 1 (MCP-1), and intercellular adhesion molecule 1 (ICAM-1) in ischaemia reperfusion (IR) gut. Reverse transcription-polymerase chain reaction (RT-PCR) was performed to analyse the relative expression of Egr-1 in the IR (IR) and sham operation (sham) gut of the 30/60 minute (30'/60') IR and 45/60 minute (45'/60') IR groups (A). Weak expression of Egr-1 mRNA was detected in control jejunum (control) and sham operation jejunum. A marked increase in Egr-1 mRNA expression in the 30/60 minute IR jejunum and a moderate increase in Egr-1 mRNA expression in the 45/60 minute IR jejunum were detected. Relative expression of Egr- 1 to $\beta$-actin was determined in each group and is shown next to the gel picture of RT-PCR. *Significant difference between the IR and sham jejunum. Protein levels of Egr-1, RANTES, ICAM-1, and MCP-1 in the jejunum of different IR groups were analysed by western blotting (B). Actin was used as a loading control for each lane. Elevated levels of Egr-1, RANTES, and MCP-1 were detected in the $45 / 60$ minute IR jejunum. Immunoreactivity of ICAM-1 was detected in villi (C), but only expressed in the $45 / 60$ minute IR jejunum in endothelial cells (arrows) of blood capillaries.

sham operation jejunum were analysed using western blotting. Levels of MPO were increased markedly in the 30/ 60 minute and 45/60 minute IR jejunum while the amount of MPO in the sham operation jejunum was negligible (fig 2C). Similar intensities of actin were observed in all lanes, indicating that equivalent amounts of protein extracts of each sample were analysed. RT-PCR analysis revealed similar levels of MPO mRNA in both sham controls and IR groups (fig 2D) suggesting that MPO induction in our IR model was regulated at the translation level.

\section{Expression of mRNA and protein of Egr-1 gene in IR gut}

To investigate if upregulation of Egr-l of resident macrophages is crucial for gut IR injury, a kinetic study of Egr-1 expression in the IR jejunum was performed. Relative expression levels of Egr-1 mRNA to $\beta$-actin mRNA were determined in IR jejunum, sham operation jejunum, and control jejunum. The relative expression level of Egr-I mRNA increased by 3.9-fold in the 30/60 minute IR jejunum

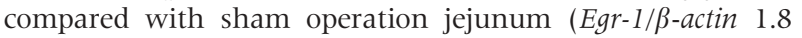
(0.19):0.46 (0.29); IR:sham; $\mathrm{p}<0.01$ ) and by threefold in the $45 / 60$ minute IR jejunum compared with the sham jejunum (Egr-1/B-actin 0.75 (0.23):0.25 (0.05); IR:sham; p>0.05) (fig 3A). The increase in the relative level of Egr-1 mRNA in the 30/60 minute IR jejunum over the sham jejunum reached statistical significance $(\mathrm{p}<0.01)$. Levels of Egr-1 protein and protein products of Egr-l downstream target genes in the IR jejunum were analysed by western blotting (fig 3B). A marked increase in Egr-1, RANTES, and MCP-1 was detected only in the 45/60 minute IR jejunum, and a slight increase in expression of ICAM-1 was also detected in the 45/60 minute IR jejunum. Immunohistochemistry showed expression of ICAM-1 at endothelial cells of the blood capillaries in the 45/60 minute IR jejunum (fig 3C).

\section{Subcellular distribution of Egr-1 in resident macrophages of IR jejunum}

Transcription factor Egr- 1 is translocated into the nucleus to induce expression of its target genes. Subcellular distribution of Egr-1 in the sham and IR jejunum was investigated by immunohistochemistry (fig 4). In the sham operation group, only a few cells at the mucosal epithelium were stained for Egr-1 (fig 4A). In the 30/60 minute IR jejunum, clear Egr-1 immunopositive cells were detected inside the villi, and Egr-1 protein was mainly localised in the cytoplasm (fig 4B). A larger number of more intensely stained Egr-1 immunopositive cells were localised in the villi of the 45/60 minute IR jejunum, and Egr-1 protein was found mainly in the nucleus (fig 4C). Colocalisation studies revealed in 45/60 minute IR injury section that ED2 immunopositive macrophages were immunoreactive for Egr-l (fig 4D). Western blotting of the nuclear and cytoplasmic fractions of IR jejunum also revealed the presence of nuclear Egr-1 in the 45/60 minute IR group (fig 4E).

Effect of resident macrophage depletion on IR injury To determine if $\mathrm{Cl}_{2} \mathrm{MBP}$ is effective in the depletion of resident macrophages in the gut, $\mathrm{Cl}_{2} \mathrm{MBP}$ liposome and control liposome injected rats were sacrificed three days postinjection, and their gastrointestinal tracts processed for immunohistochemistry to localise the resident macrophages (ED2 immunopositive) and total macrophages (EDl immunopositive) in the liver and gut. Resident macrophages were markedly decreased both in the liver and gut of $\mathrm{Cl}_{2} \mathrm{MBP}$ liposome treated rats (fig 5A-D). The percentage of resident macrophages to total macrophages decreased from $41.3 \%$ (4.9) in control liposome treated guts to $1.8 \%(0.8)$ in the $\mathrm{Cl}_{2} \mathrm{MBP}$ liposome treatment group (fig 5). Levels of expression of Egr-1, cytokines, and adhesion molecule ICAM-1 were analysed in the jejunum of control and $\mathrm{Cl}_{2} \mathrm{MBP}$ liposome 

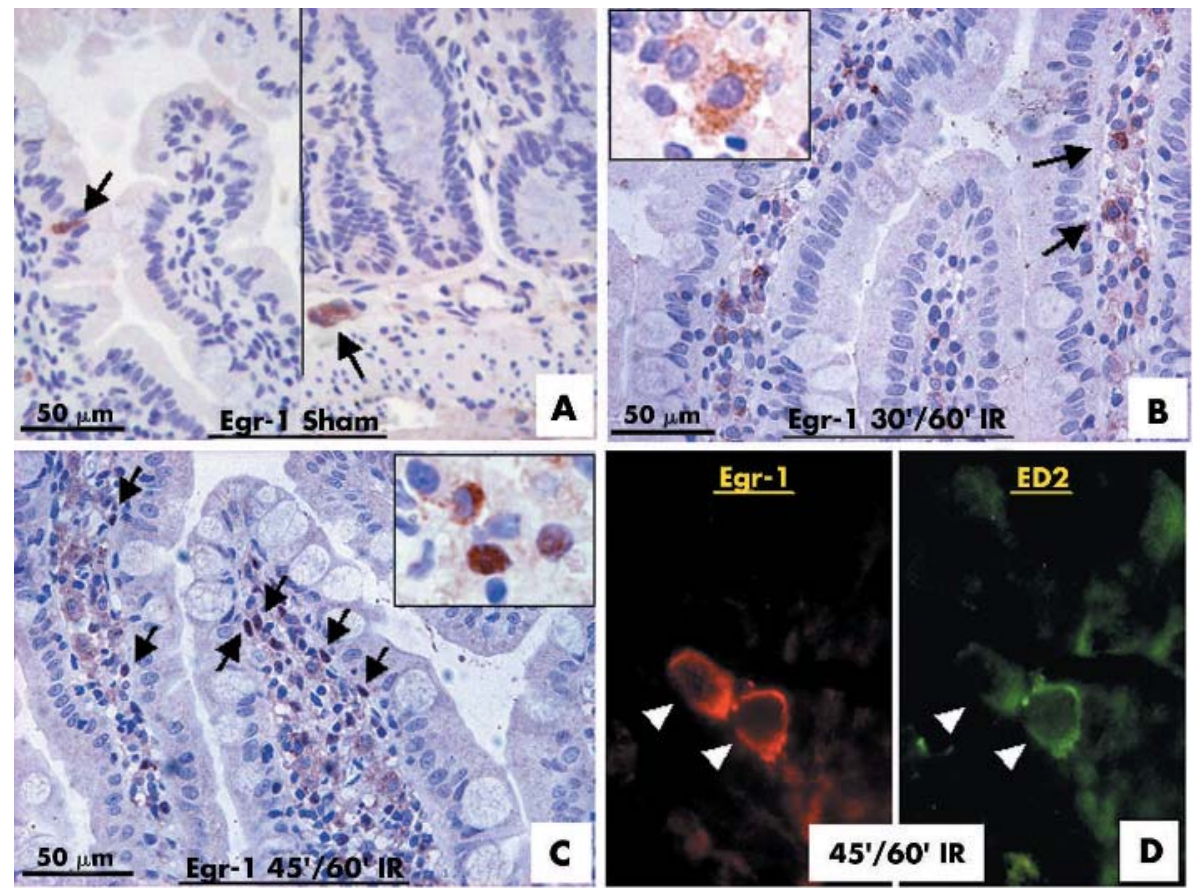

\begin{tabular}{|c|c|c|}
\cline { 2 - 3 } \multicolumn{1}{c|}{} & Cytoplasm + & Nuclear + \\
\hline Sham group & 0.1 & 0 \\
\hline $\begin{array}{c}30 / 60 \mathrm{IR} \\
\text { group }\end{array}$ & $9.5(1.82)$ & $1.4(0.35)$ \\
\hline $\begin{array}{c}45 / 60 \mathrm{IR} \\
\text { group }\end{array}$ & $5.0(0.35)$ & $7.4(0.99)$ \\
\hline
\end{tabular}

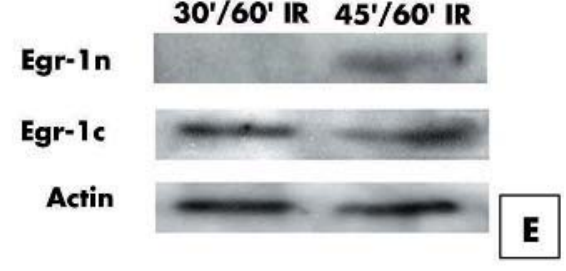

Figure 4 Resident macrophages expressed a high level of early growth response factor 1 (Egr-1) in the ischaemia reperfusion (IR) jejunum. In the sham operation jejunum, Egr-1 immunoreactivity was not detected in villi and only a few cells at the mucosal epithelium showed Egr-1 expression (A). A few Egr-1 immunopositive cells (arrows) were localised in the villi of the $30 / 60$ minute $\left(30^{\prime} / 60^{\prime}\right)$ IR jejunum (B), and the Egr-1 protein was in the cytoplasm (inset). A large number of Egr-1 immunopositive cells (arrows) were localised in the damaged villi of the 45/60 minute $\left(45^{\prime} / 60^{\prime}\right.$ ) IR jejunum (C), and the Egr-1 protein was mainly in the nuclei (inset). Cells showing cytoplasmic Egr-1 staining (Cytoplasmic + ) and cells showing nuclear Egr-1 staining (Nuclear + ) in villi of different treatment groups were counted and tabulated as shown. An immunofluorescent colocalisation study using antiEgr-1 antibody and ED2 antibody (D) in 45/60 minute IR injury gut indicated that ED2 immunopositive resident macrophages were also immunopositive for Egr-1 (arrowheads). Distribution of Egr-1 protein in the nuclear and cytoplasmic fractions of jejunum of 30/60 minute and 45/60 minute IR groups were investigated with western blotting. Egr-1 was detected in the nuclear fraction (Egr-1n) of 45/60 minute IR jejunum while Egr-1 was detected in cytoplasmic fractions (Egr-1c) in both groups (E). Actin was used as a loading control for each lane.

treatment groups. mRNA and protein levels of Egr-1 were reduced in the $\mathrm{Cl}_{2} \mathrm{MBP}$ liposome treatment group (fig 5E, F). Levels of RANTES and MCP-l were reduced after $\mathrm{Cl}_{2} \mathrm{MPB}$ treatment (fig 5F). In contrast, only a slight reduction in ICAM- 1 level was observed in the $\mathrm{Cl}_{2} \mathrm{MBP}$ liposome treatment group (fig 5F). To examine if the depletion of gut resident macrophages can alleviate mucosal damage after IR, we performed histological analysis of the 45/60 minute IR jejunum of $\mathrm{Cl}_{2} \mathrm{MBP}$ liposome and control liposome treated rats. Sloughing of mucosal epithelium and villi destruction were detected in the control jejunum (fig 6A). In contrast, the mucosa appeared intact and epithelial sloughing was rarely observed in the $\mathrm{Cl}_{2} \mathrm{MBP}$ liposome treated jejunum (fig 6B). The percentage of damaged villi to total villi was reduced from $34.2 \%$ (2.5) in the control liposome treated jejunum to $5.2 \%$ (2.2) in the $\mathrm{Cl}_{2} \mathrm{MBP}$ liposome treated jejunum.

\section{DISCUSSION}

In this study, we have reported that mucosal damage occurs before PMN infiltration in an IR injured gut in a rat model. IR induced mucosal damage was correlated with activation of gut resident macrophages and upregulation of MPO and
Egr-1 of resident macrophages. Depletion of gut resident macrophages by $\mathrm{Cl}_{2} \mathrm{MBP}$ significantly reduced mucosal damage. The present study provides evidence suggesting that activated resident macrophages play a key role in early IR damage to the gut.

Sloughing of mucosal epithelium and ulceration of villi have been recognised as early histological landmarks of IR gut injury, ${ }^{2}$ and these were observed in 45/60 minute IR gut in our model. However, only a few PMN were found at the damaged villi and at the base of the crypt although clear mucosal damage was evident at the IR jejunum. PMN were localised at the base of the crypt in the 60/60 minute IR gut. Massive PMN infiltration was detectable in the damaged area after three hours of reperfusion. This finding is in line with previous reports that PMN infiltration is detectable in damaged tissues 4-6 hours after IR. ${ }^{31-33}$ Taken together, these findings suggest that early mucosa damage in IR injury is not attributable to PMN. Interaction between ICAM-1 on endothelial cells and LFA-1 on PMN is a prerequisite for PMN infiltration. ${ }^{34}$ ICAM-1 expression of endothelial cells was first observed at 45/60 minute IR gut, which suggested that IR induced mucosal damage began prior to PMN 

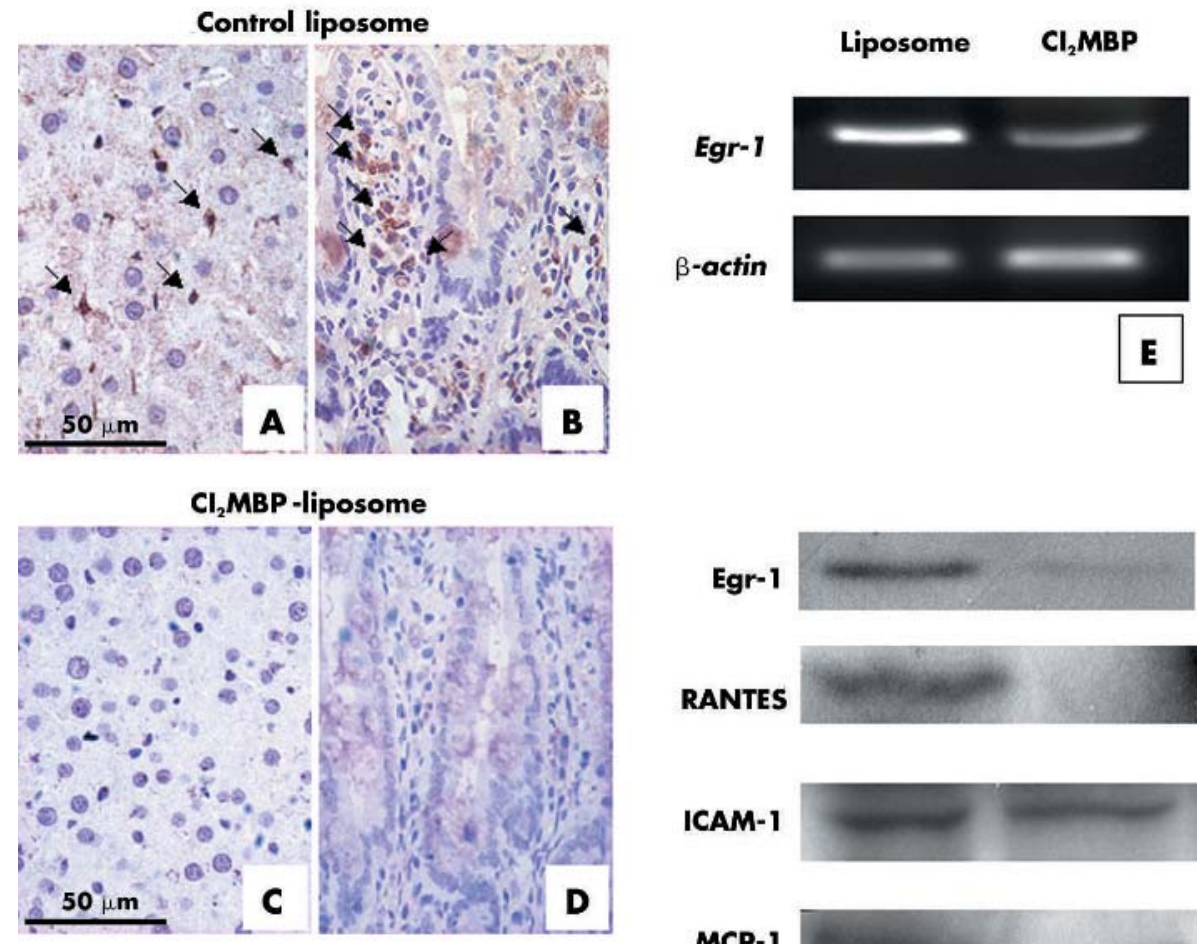

\begin{tabular}{|l|c|}
\hline Treatment & ED2/ED1 \\
\hline $\begin{array}{l}\text { Control } \\
\text { liposome }\end{array}$ & $41.3 \%(4.9 \%)$ \\
\hline $\begin{array}{l}\mathrm{Cl}_{2} \mathrm{MBP} \\
\text { liposome }\end{array}$ & $1.8 \%(0.8 \%)$ \\
\hline
\end{tabular}

ICAM-1

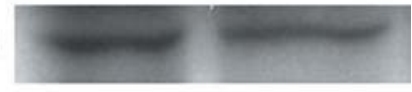

MCP-1

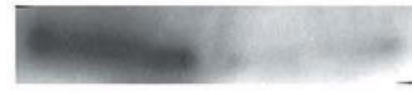

Actin

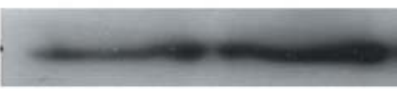

Figure 5 Intraperitoneal injection of dichloromethylene bisphosphonate $\left(\mathrm{Cl}_{2} \mathrm{MBP}\right)$ depleted resident macrophages from the gut and liver. Sections from the liver $(A, C)$ and jejunum $(B, D)$ of rats treated with control liposome or $\mathrm{Cl}_{2} \mathrm{MBP}$ liposome were immunostained with ED2 antibody to localise resident macrophages. ED2 immunopositive resident macrophages (arrows) were localised in the liver (A) and jejunum (B) of control liposome treated rats but were undetectable in the liver $(C)$ and jejunum (D) of $\mathrm{Cl}_{2} \mathrm{MBP}$ liposome treated rats. The percentage of ED2 immunopositive resident macrophages versus EDI immunopositive total macrophages in the control liposome treated and $\mathrm{Cl}_{2} \mathrm{MBP}$ liposome treated jejunum was determined and tabulated as shown (number of views studied for reach group =30). Expression of early growth response factor 1 (Egr-1) mRNA in the control liposome treatment group and the $\mathrm{Cl}_{2} \mathrm{MBP}$ treatment group was analysed by reverse transcription-polymerase chain reaction (E). Levels of Egr-1, cytokines, and intercellular adhesion molecule 1 (ICAM-1) in the jejunum of the control liposome treatment group and the $\mathrm{Cl}_{2} \mathrm{MBP}$ treatment group were analysed by western blotting (F). Reduction of Egr-1, RANTES, and monocyte chemoattractant protein 1 (MCP-1) was observed in the $\mathrm{Cl}_{2} \mathrm{MBP}$ treatment group. In contrast, only a slight reduction in the level of ICAM-1 was observed in the Cl2MBP treatment group compared with that in the control liposome treatment group.

infiltration, and corroborates a report that anti-PMN antiserum fails to prevent tissue damage after IR in rats. ${ }^{33}$ Our results also suggest the importance of ischaemia in the priming of gut damage in which longer ischaemia results in more damage after reperfusion.

Elevated tissue MPO enzyme activity has been frequently used as an indication of PMN infiltration in IR injuries. ${ }^{18}$ However, monocytes and macrophages also express MPO, which indicates that elevated tissue MPO activity is not necessarily derived from infiltrated PMN. Furthermore, IR induced mucosal damage began prior to PMN infiltration, which indicated that elevated MPO activity in the early phase of IR gut injury was not attributed to PMNs. Immunostaining using an anti-MPO antibody and tissue macrophage marker (ED2) revealed that resident macrophages in IR guts expressed MPO enzyme. Activated macrophages in vitro ${ }^{14}$ have already been shown to express a high level of MPO activity. Indeed, resident macrophages in the IR jejunum expressed activated macrophage markers LFA- $1^{35}$ and iNOS, ${ }^{36}$ suggesting that resident macrophages were activated in the IR gut. Elevated expression of MPO in resident macrophages and increased tissue MPO levels were detected in the 30/60 minute IR jejunum, and mucosal damage was evident in the 45/60 minute IR jejunum. MPO catalyses the production of $\mathrm{OCl}^{-}$and chloramines which have already been demonstrated to cause gastric mucosal cell apoptosis and cell sloughing. ${ }^{16}{ }^{17}$ Elevated MPO activity is associated with pulmonary injury, renal glomerular damage, and the initiation of atherosclerotic lesions. ${ }^{37}$ We failed to detect mucosal damage in the jejunum after prolonged ischaemia (two hours; unpublished data). This suggests that the oxygen burst during reperfusion triggers the cascade of cellular activations and molecular events leading to IR damage.

To prove that resident macrophages were involved in gut IR injury, we compared IR damage in $\mathrm{Cl}_{2} \mathrm{MBP}$ liposome and control liposome treated rats. $\mathrm{Cl}_{2} \mathrm{MBP}$ liposome can 


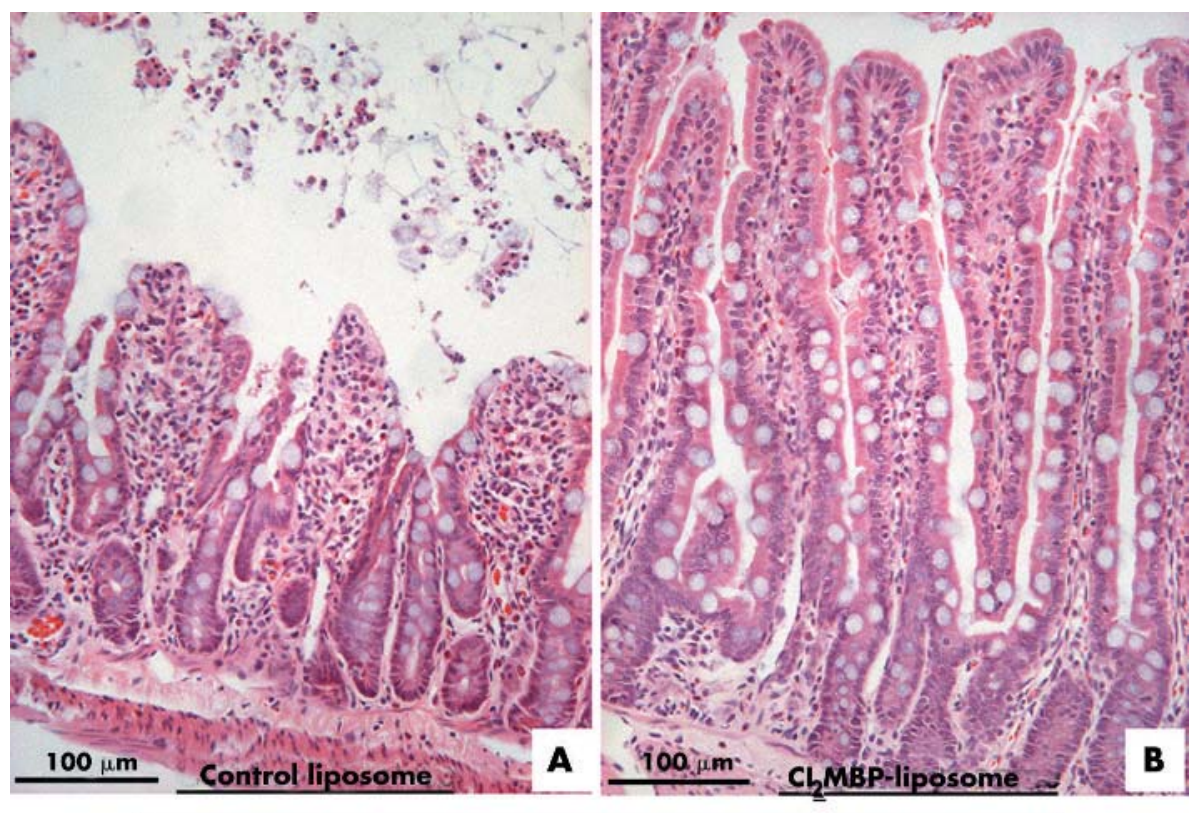

Figure 6 Ischaemia reperfusion (IR) induced mucosal damage was markedly reduced in dichloromethylene bisphosphonate $\left(\mathrm{Cl}_{2} \mathrm{MBP}\right)$ liposome treated jejunum. Haematoxylin and eosin staining of sections of the 45 / 60 minute IR jejunum of control liposome treated rats $(A)$ and $\mathrm{Cl}_{2} \mathrm{MBP}$ liposome treated rats (B) are shown. The percentage of damaged villi versus total villi in the control liposome and $\mathrm{Cl}_{2} \mathrm{MBP}$ liposome treated rats was determined and tabulated as shown.

\begin{tabular}{|l|c|}
\hline Treatment & Damage villi / total villi \\
\hline Control liposome & $34.2 \%(2.5 \%)$ \\
\hline $\mathrm{Cl}_{2}$ MBP-liposome & $5.2 \%(2.2 \%)$ \\
\hline
\end{tabular}

specifically deplete resident macrophages in the gut without changing PMN activity. ${ }^{38}$ The percentage of resident macrophages (ED2 immunopositive) to total macrophages (EDl immunopositive) in the gut decreased from $41.3 \%$ in control liposome treated guts to only $1.8 \%$ in the $\mathrm{Cl}_{2} \mathrm{MBP}$ liposome treated gut. Only ED2 immunopositive resident macrophages were depleted in $\mathrm{Cl}_{2} \mathrm{MBP}$ treated rats indicating that $\mathrm{Cl}_{2} \mathrm{MBP}$ was specific in the depletion of resident macrophages. The degree of IR induced mucosal damage and villi destruction was markedly reduced in the $\mathrm{Cl}_{2} \mathrm{MBP}$ liposome treated jejunum compared with the control jejunum. Mucosal damage was not detectable in resident macrophages depleted rats even after three hours of reperfusion, and very few PMN were localised at the villi. Our findings on IR injury to resident macrophage depleted guts suggest that resident macrophages contribute to the initiation of mucosal damage in IR.

Colocalisation analysis established that resident macrophages in the damaged area expressed Egr-1. Activation of resident macrophages induces mRNA and protein expression of Egr-1 gene, and nuclear compartmentalisation of Egr-1 protein in resident macrophages. Involvement of Egr-1 in lung IR injury has been shown in mice. PMN infiltration and upregulation of cytokines, including RANTES and MCP-1, in mouse lungs after IR challenge are markedly reduced in Egr-I knockout mice and these mice show very mild IR damage in lung tissue. ${ }^{22}$ The reduction in Egr-1, RANTES, and MCP-1 after $\mathrm{Cl}_{2} \mathrm{MBP}$ treatment indicated that macrophages produced these cytokines. However, studies of gut IR in Egr-l knockout mice could further clarify the Egr-l function in the onset of gut IR damage. Our data suggest the PMN infiltration by Egr-1 upregulation is not directly implicated in the onset of gut IR injury. Reduction of PMN infiltration in $\mathrm{Cl}_{2} \mathrm{MBP}$ treated guts after IR is likely to be an indirect effect of reduced macrophage derived cytokines that induce PMN infiltration. $^{40}$

In summary, activated resident macrophages in IR gut express a high level of MPO, Egr-1, and proinflammatory cytokines. Elevated MPO activity catalyses the production of reactive free radicals during reperfusion, causing initial mucosal epithelial cell death. Accumulation of proinflammatory cytokines induces PMN infiltration, resulting in further tissue damage. Further exploration of potential therapeutic intervention for IR damage might therefore profitably be directed towards resident macrophages, as they have the capacity to prevent initial mucosal destruction and arrest subsequent tissue damage.

\section{Authors' affiliations}

Y Chen, V C H Lui, P K H Tam, Division of Paediatric Surgery,

Department of Surgery, University of Hong Kong Medical Centre, Queen Mary Hospital, University of Hong Kong, China

N V Rooijen, Department of Cell Biology, Division of Histology, Faculty of Medicine, Free University, Amsterdam, the Netherlands

\section{REFERENCES}

1 Jrvinen O, Laurikka J, Salenius JP, et al. Acute intestinal ischaemia. A review of 214 cases. Ann Chir Gynaecol 1994;83:22-5.

2 Kong SE, Blennerhassett $L R$, Heel KA, et al. Ischaemia-reperfusion injury to the intestine. Aust N Z J Surg 1998;68:554-61.

3 Jaeschke $\mathbf{H}$. Mechanisms of reperfusion injury after warm ischemia of the liver. J Hepatobiliary Pancreat Surg 1998;5:402-8.

4 Jordan JE, Zhao ZQ, Vinten-Johansen J. The role of neutrophils in myocardial ischemia-reperfusion injury. Cardiovasc Res 1999;43:860-78.

5 Turnage RH, Guice KS, Oldham KT. Pulmonary microvascular injury following intestinal reperfusion. New Horiz 1994;2:463-75.

6 Kohtani T, Abe Y, Sato $M$, et al. Protective effects of anti-neutrophil antibody against myocardial ischemia/reperfusion injury in rats. Eur Surg Res 2002;34:313-20. 
7 Andoh A, Fujiyama $Y$, Araki $Y$, et al. Role of complement activation and mast cell degranulation in the pathogenesis of rapid intestinal ischemia/reperfusion injury in rats. Digestion 2001;63(Suppl 1):103-7.

8 Nagaoka I, Kaneko H, Yamashita T. Inhibition of the accumulation of macrophages and the generation of macrophage chemotactic activity by dexamethasone in concanavalin A-induced peritonitis of mice. Agents Actions 1988;25:156-63

9 Hudig D, Redelman D, Minning L, et al. Inhibition of human lymphocyte natural cytotoxicity and antibody-dependent cell-mediated cytotoxicity by $\mathrm{K}$ $76 \mathrm{COONa}$ a reagent that blocks complement activity. J Immunol 1984; 133:408-14.

10 Coates NJ, McColl SR. Production of chemokines in vivo in response to microbial stimulation. J Immunol 2001;166:5176-82.

11 Askari AT, Brennan ML, Zhou X, et al. Myeloperoxidase and plasminogen activator inhibitor 1 play a central role in ventricular remodeling after myocardial infarction. J Exp Med 2003;197:615-24.

12 Zimmerman BJ, Grisham MB, Granger DN. Role of oxidants in ischemia/ reperfusion-induced granulocyte infiltration. Am J Physiol 1990;258:G185-90.

13 Heinecke JW, Li W, Daehnke HLR, et al. Dityrosine, a specific marker of oxidation, is synthesized by the myeloperoxidase-hydrogen peroxide system of human neutrophils and macrophages. J Biol Chem 1993;268:4069-77.

14 Rodrigues MR, Rodriguez D, Russo M, et al. Macrophage activation includes high intracellular myeloperoxidase activity. Biochem Biophys Res Commun 2002;292:869-73.

15 Duffield JS. The inflammatory macrophage: a story of Jekyll and Hyde. Clin Sci (Lond) 2003; 104:27-38

16 Naito Y, Yoshikawa T, Fujii T, et al. Monochloramine-induced cell growth inhibition and apoptosis in a rat gastric mucosal cell line. J Clin Gastroenterol 1997;25(suppl 1):S179-85.

17 Suzuki H, Seto K, Mori M, et al. Monochloramine induced DNA fragmentation in gastric cell line MKN45. Am J Physiol 1998;275:G712-16.

18 De Greef KE, Ysebaert DK, Ghielli M, et al. Neutrophils and acute ischemiareperfusion injury. J Nephrol 1998;11:110-22.

19 Chiu CJ, McArdle AH, Brown R, et al. Intestinal mucosal lesion in low-flow states. I. A morphological, hemodynamic, and metabolic reappraisal. Arch Surg 1970;101:478-83.

20 Hierholzer C, Kalff JC, Audolfsson G, et al. Molecular and functional contractile sequelae of rat intestinal ischemia/reperfusion injury. Transplantation 1999;68:1244-54.

21 Beckmann AM, Wilce PA. Egr transcription factors in the nervous system. Neurochem Int 1997:31:477-510.

22 Yan SF, Fujita T, Lu J, et al. Egr-1, a master switch coordinating upregulation of divergent gene families underlying ischemic stress. Nat Med 2000;6:1355-61.

23 Aebert $\mathrm{H}$, Cornelius T, Ehr T, et al. Expression of immediate early genes after cardioplegic arrest and reperfusion. Ann Thorac Surg 1997;63:1669-75.
24 Brand T, Sharma HS, Fleischmann KE, et al. Proto-oncogene expression in porcine myocardium subjected to ischemia and reperfusion. Circ Res 1992;71:1351-60.

25 Bonventre JV, Sukhatme VP, Bamberger $M$, et al. Localization of the protein product of the immediate early growth response gene, Egr-1, in the kidney after ischemia and reperfusion. Cell Regul 1991;2:251-60.

26 Ouellette AJ, Malt RA, Sukhatme VP, et al. Expression of two "immediate early" genes, Egr-1 and c-fos, in response to renal ischemia and during compensatory renal hypertrophy in mice. J Clin Invest 1990;85:766-71.

27 Van Rooijen N, Sanders A. Liposome mediated depletion of macrophages: mechanism of action, preparation of liposomes and applications. J Immunol Methods 1994; 174:83-93.

28 van den Berg TK, Dopp EA, Dijkstra CD. Rat macrophages: membrane glycoproteins in differentiation and function. Immunol Rev 2001;184:45-57.

29 Barbe E, Damoiseaux JG, Dopp EA, et al. Characterization and expression of the antigen present on resident rat macrophages recognized by monoclonal antibody ED2. Immunobiology 1990;182:88-99.

30 Deryckere F, Gannon F. A one-hour minipreparation technique for extraction of DNA-binding proteins from animal tissues. Biotechniques 1994;16:405.

31 Koike K, Moore EE, Moore FA, et al. CDI $1 \mathrm{~b}$ blockade prevents lung injury despite neutrophil priming after gut ischemia/reperfusion. J Trauma 1995:39:23-7.

32 Schoenberg MH, Poch B, Younes M, et al. Involvement of neutrophils in postischaemic damage to the small intestine. Gut 1991;32:905-12.

33 Simpson R, Alon R, Kobzik L, et al. Neutrophil and nonneutrophil-mediated injury in intestinal ischemia-reperfusion. Ann Surg 1993;218:444-53.

34 Olanders K, Sun Z, Borjesson A, et al. The effect of intestinal ischemia and reperfusion injury on ICAM-1 expression, endothelial barrier function, neutrophil tissue influx, and protease inhibitor levels in rats. Shock 2002;18:86-92.

35 Kalff JC, Turler A, Schwarz NT, et al. Intra-abdominal activation of a local inflammatory response within the human muscularis externa during laparotomy. Ann Surg 2003;237:301-15.

36 Hori M, Kita M, Torihashi S, et al. Upregulation of iNOS by COX-2 in muscularis resident macrophage of rat intestine stimulated with LPS. Am J Physiol Gastrointest Liver Physiol 2001 ;280:G930-8.

37 Klebanoff SJ. Myeloperoxidase. Proc Assoc Am Physicians 1999;1 1 1:383-9.

38 Fryer J, Grant D, Jiang J, et al. Influence of macrophage depletion on bacterial translocation and rejection in small bowel transplantation. Transplantation 1996;62:553-9.

39 Schmidt-Weber CB, Rittig M, Buchner E, et al. Apoptotic cell death in activated monocytes following incorporation of clodronate-liposomes. J Leukoc Biol 1996;60:230-44.

40 Ebe $Y$, Hasegawa G, Takatsuka H, et al. The role of Kupffer cells and regulation of neutrophil migration into the liver by macrophage inflammatory protein-2 in primary listeriosis in mice. Pathol Int 1999;49:519-32.

\section{EDITOR'S QUIZ: GI SNAPSHOT}

\begin{abstract}
Answer
From question on page 1771

Terminal ileoscopy revealed multiple red spots and raised cherry red bumps $3-5 \mathrm{~mm}$ in diameter, with a mosaic pattern of the mucosa around their base. Biopsies revealed oedema in the mucosa and arteriolar dilations that sometimes contained fibrin thrombi and were compatible with hypertensive portal ileopathy. Videocapsule endoscopy revealed diffuse involvement of the small intestine, with increasingly severe vascular lesions from the proximal jejunum up to the distal ileum where these particular raised red bumps were exclusively located. All of the lesions were attributed to portal hypertension enteropathy and considered to be the cause of the recurrent gastrointestinal bleeding. The patient was given $80 \mathrm{mg} /$ day propranolol and six months later he no longer exhibited either melena or a relapsing decrease in haemoglobin.

This case exhibited a novel endoscopic appearance of portal hypertensive enteropathy characterised by raised red bumps in the ileum, combined with particular histological features similar to those of gastric antral vascular ectasia.
\end{abstract}

doi: $10.1136 /$ gut.2003.034611 